\section{Early life infections and the risk of hay fever}

\author{
Victoria S Hammersley and *Aziz Sheikh ${ }^{b}$ \\ a Research Fellow, ${ }^{b}$ Professor of Primary Care Research \& \\ Development, Allergy \& Respiratory Research Group, \\ Division of Community Health Sciences: GP Section, \\ University of Edinburgh, UK
}

*Corresponding author: 20 West Richmond St, Edinburgh, EH8 9DX, Scotland, UK. Tel: +44 (0)131 6514151 Fax: +44 (0)131 6509119 E-mail: aziz.sheikh@ed.ac.uk

\section{Bremner SA, Carey IM, DeWilde S, et al. Infections presenting for clinical care in early life and later risk of hay fever in two UK birth cohorts. Allergy 2008;63:274-83.}

Does early life exposure to infections offer protection against the risk of developing atopic allergic diseases? Almost two decades on from the initial formulation of the 'hygiene hypothesis', ${ }^{1}$ and despite a considerable body of epidemiological and basic science research investigating this relationship, there is still no clear answer to this intriguing question. ${ }^{2,3}$ Whilst this paper by Bremner et al does not answer the question definitively, it does represent a welcome contribution to the evidence base refuting $a$ simplecausal relationship between early life infection and risk of developing IgE-mediated conditions.

Bremner and colleaguesundertook secondary analyses of data from two very large UK primary care databases: the General Practice Research Database (GPRD) and the Doctors Independent Network (DIN). Using a nested case-control study design ${ }^{4}$ they investigated the association between a variety of childhood infections recorded in the GP records and subsequent development of hay fever - the archetypal IgEmediated condition.

Using primary care data in this way allows researchers to study a broad range of common and rare infections presenting to GPs in early childhood, something that would not easily be achieved through primary research studies. Thus a key strength of this study was the investigation of 30 infections, many of which have never previously been studied in this context. Another advantage of using routine data in this way was a longer follow-up than is often possible in cohort studies: all children in these studies had at least five years of continuous follow-up.

Confounding is a key consideration in case-control studies and a major strength of this work was the attempt to control, and adjust for, a range of potentially relevant factors. For example, each case of hay fever had one control matched for GP, sex, birth month and follow-up to at least the date of diagnosis of hay fever. Also important is that regular attenders to general practice are more likely to have diagnoses recorded in their medical records and are thus more likely to have a recorded diagnosis of hay fever if they experience suggestive symptoms. This could therefore lead to a spurious positive relationship between infections and hay fever. To their credit, the authors rigorously explored six possible adjustments for consultation frequency confounding, as well as adjusting for sibship size and socio-economic factors.

Unadjusted odds ratios (ORs) for hay fever amongst children presenting with infection compared with those not presenting were positive for 20 infections in GPRD and 23 infections in DIN. A strong positive association was also seen between behavioural problems and hayfever, an internal control condition. Following adjustment, however, most of the positive odds ratios disappeared, and of those that remained, none reached statistical significance. Further anatyses show the importance of consultation frequency as a confounding variable indicated by the strong positive relationship between hay fever and the number of consultations in years 2-5: those with over 10 consultations had an OR of hay fever of 8.5 in GPRD and 9.6 in DIN compared with those with only one consultation.

Sibship size was explored within the GPRD, and although the risk of hay fever fell with increasing number of older siblings - in keeping with the original hypothesis - this could not be explained by consultations for infection. ${ }^{1}$

The bottom line from these analyses is that the relationship between birth order and risk of hay fever is robust, but that childhood infections are an unlikely explanation. The search for an adequate explanation for this remarkably consistent observation thus continues...

\section{Conflict of interest declaration}

Professor Aziz Sheikh is an Assistant Editor of the PCRJ, but was not involved in the editorial review of, nor the decision to publish, this article.

\section{References}

1. Strachan D. Hay fever, hygiene, and household size. Br Med J 1989;299:1259-60.

2. Strachan DP. Family size, infection and atopy: the first decade of the 'hygiene hypothesis'. Thorax 2000;55(Suppl 1):S2-10.

3. Sheikh A, Strachan DP. The hygiene theory: fact or fiction? Curr Opin Otolaryngol Head Neck Surg 2004;12(3):232-6

4. Rothman KJ, Greenland S. Modern epidemiology. 2nd ed. ed: Philadelphia: Lippincott-Raven, 1998.

doi:10.3132/pcrj.2008.00034 